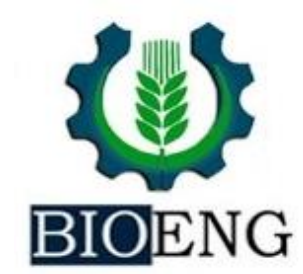

\section{OTIMIZAÇÃO DO USO DE INSUMOS EM SISTEMA DE INTEGRAÇÃO LAVOURA-PECUÁRIA-FLORESTA COM FERRAMENTAS DE AGRICULTURA DE PRECISÃO}

\section{A. C. de C. Bernardi ${ }^{1 *}$; N. Laurenti ${ }^{2}$; G. M. Bettiol ${ }^{3}$; P. P. A. de Oliveira $^{1}$; T. C. Alves ${ }^{1}$; A. de F. Pedroso ${ }^{1}$; S. N. Esteves ${ }^{1}$, J. R. M. Pezzopane ${ }^{1}$}

${ }^{1}$ Embrapa Pecuária Sudeste, São Carlos, SP, Brasil

${ }^{2}$ UNICEP, Centro Universitário Central Paulista, São Carlos, SP, Brasil

${ }^{3}$ Embrapa Cerrados, Planaltina - DF

Article history: Received 03 October 2019; Received in revised form 04 November 2019; Accepted 07 November 2019; Available online 05 December 2019.

\title{
RESUMO
}

O conhecimento da variabilidade espacial das propriedades do solo é útil para o uso racional dos insumos, como na aplicação localizada de calcário e fertilizantes nos sistemas de integração lavoura-Pecuária-Floresta (ILPF). A agricultura de precisão (PA) é a ferramenta para melhorar a eficiência do uso destes insumos. O objetivo desta pesquisa foi avaliar a aplicação calagem e de fertilizante fosfatado a taxas variáveis em um sistema ILPF. O estudo de campo foi realizado em uma área de 30 ha da Embrapa Pecuária Sudeste, em São Carlos, SP. As amostras de solo foram coletadas a 0-0,2 $\mathrm{m}$ de profundidade, e cada amostra representou um piquete. A variabilidade espacial das propriedades do solo e das necessidades específicas de calcário e fertilizantes foram modeladas. Os resultados mostraram que a análise espacial das necessidades de calagem e adubação podem fornecer ferramentas de gestão para o manejo de pastagens. E a tecnologia da aplicação de calcário e fertilizante fosfatado à taxa variável pode ser utilizada como ferramenta de correção e adubação do solo levando à maior homogeneidade dos atributos químicos do solo.

Palavras-chave: geoprocessamento, taxa variável, sistemas integrados, calagem, adubação fosfática.

\section{INPUTS USE OPTIMIZATION OF A CROP-LIVESTOCK-FOREST INTEGRATION SYSTEM WITH PRECISION AGRICULTURE TOOLS}

\begin{abstract}
Knowledge on spatial variability of soil properties is useful for the rational use of inputs, as in the site-specific application of lime and fertilizer in the crop-livestock-forest integrated systems (CLFIS). Precision agriculture (PA) is the tool to improve the efficiency of use of these issues. The objective of this research was to evaluate liming and fertilizer variable rate application in CLFIS. The field study was carried out in a 30-ha area at Embrapa Pecuária Sudeste in São Carlos, SP, Brazil. Soil samples were collected at 0-0.2 m depth, and each sample represented a paddock. The spatial variability of soil properties and site-specific liming and fertilizer need were modeled by inverse distance weighting (IDW) technique. The results showed that spatial analysis of liming and fertilization needs can provide management tools for pasture management. And variable rate lime and phosphate fertilizer application
\end{abstract}

\footnotetext{
*alberto.bernardi@embrapa.br
} 
technology can be used as a soil correction and fertilization tool leading to greater homogeneity of soil chemical attributes.

Keywords: geoprocessing, variable rate, integrated systems, lime, phosphorus fertilizer.

\section{INTRODUÇÃO}

Os Sistemas de Integração LavouraPecuária-Floresta (ILPF) têm sido utilizados como uma estratégia de intensificação agrícola sustentável que integra atividades anuais de cultivo, árvores e pecuária na mesma área e na mesma safra (BALBINO et al., 2011). Entre os fatores controláveis que determinam a produtividade e a qualidade das culturas, está o manejo da fertilidade do solo. Nos solos intemperizados, de baixa fertilidade e ácidos da região tropical brasileira (BERNARDI et al. 2012) o fornecimento balanceado de nutrientes é importante para obter-se altos rendimentos e é essencial para manter alta qualidade e rendimentos rentáveis no ILPF. O manejo da fertilidade do solo pode ter grande impacto na produtividade e qualidade das pastagens (CANTARELLA et al., 2002).

A análise química é essencial para avaliar a fertilidade do solo. Pois, com a interpretação dos resultados é possível realizar manejo químico do solo de maneira eficiente e econômica (RAIJ, 1991). Além disso, o balanço de nutrientes no sistema solo-planta pode ser considerado um indicador da sustentabilidade do uso agrícola do solo. No entanto, a gestão da fertilidade do solo, sem levar em conta a variação espacial pode afetar diretamente a produtividade e a qualidade ambiental (BERNARDI et al., 2015).

Considerando que a agricultura de precisão (AP) é um conjunto de tecnologias e práticas agrícolas para o tratamento desta variabilidade espacial, e sua implementação contribui para a gestão do sistema de produção (INAMASU et al., 2011). A AP envolve a obtenção e processamento de informações detalhadas e georreferenciadas sobre as áreas de cultivo agrícola, visando definir estratégias de manejo mais eficientes, em especial, o uso racional de insumos (BERNARDI et al., 2015).

A gestão de sistemas de produção com base nas zonas de manejo considera que as variações de relevo, solo, água e nutrientes podem ser mapeadas $\mathrm{e}$ as diferentes regiões indicadas (BERNARDI et al., 2017, 2018). Assim, cada divisão devida suas características próprias, é considerada uma unidade de gestão na qual são implementadas as aplicações de taxa variável de insumos. Nos sistemas de pastejo rotacionado, os "piquetes" podem ser considerados uma unidade de manejo (BERNARDI et al., 2017, 2018).

A tecnologia de aplicação em taxa variável de insumos, ou "Variable Rate Technology", é implementada a partir de resultados de análises químicas de amostras de solo georreferenciadas, análise da variabilidade espacial, e de mapas de fertilidade do solo e necessidades de insumos (GILLINGHAM, 2001; BERNARDI et al., 2015). Resende et al. (2014) mostrou que, a partir de mapas de atributos químicos do solo elaboram-se mapas de fornecimento de corretivos e fertilizantes em quantidades distintas para diferentes partes do talhão. Os trabalhos de Bernardi et al. (2016, 2017, 2018) e Santos et al. (2017) mostraram as possibilidades de uso em sistema de produção pecuária com base no uso de pastagens. Dessa forma, o uso de ferramentas de AP, como a amostragem de solo georreferenciada, estabelecimento de zonas de manejo e aplicação de insumos a taxa variável podem ser úteis para a gestão de sistemas ILPF. O objetivo desta pesquisa foi avaliar a aplicação calagem e de fertilizante fosfatado a taxas variáveis em um sistema ILPF. 


\section{MATERIAL E MÉTODOS}

\section{Caracterização da área de estudo}

$\mathrm{O}$ estudo foi realizado em área experimental (Figura 1) da Embrapa Pecuária Sudeste em São Carlos, Brasil $\left(21^{\circ} 57^{\prime} \mathrm{S}, \quad 47^{\circ} 50^{\prime} \mathrm{W}, \quad 860 \mathrm{~m}\right.$ alt $)$ em Latossolo vermelho-amarelo (CALDERANO et al., 1998). O clima da região (CWa) é tropical de altitude (ROLIM et al., 2007), com $1.502 \mathrm{~mm}$ de precipitação pluvial anual e médias de temperatura mínima e de temperatura máxima de $16,3^{\circ} \mathrm{C}$ (julho) e de $23^{\circ} \mathrm{C}$ (fevereiro), respectivamente.

O sistema ILPF totaliza 30 ha e inclui diferentes combinações: i) pastagem intensiva (INT) de capim Piatã (Urochloa brizantha); ii) Integração lavoura-pecuária (ILP), em que um terço da área é renovada anualmente plantando milho consorciado com capim Piatã; iii) integração florestapecuária-floresta (ILPF) plantado com Eucalyptus urograndis (GG100) em fileiras simples com espaçamento de $15 \mathrm{~m}$ e distância de $2 \mathrm{~m}$ entre árvores; iv) integração de pastagem-floresta (IPF), com capim Piatã e eucalipto; v) sistema extensivo (EXT) de capim braquiária (Urochloa decumbens). As pastagens são manejadas em sistema rotacionado com 6 dias de pastejo e 35 dias de repouso em épocas de chuvas e secas. Os piquetes são divididos com cercas elétricas em 6 subdivisões de 0,5 ha cada com 2 repetições.

\section{Amostragem do solo e recomendação de insumos}

A estratégia de amostragem do solo foi baseada na coleta de 6 sub-amostras a $0-0,2 \mathrm{~m}$ de profundidade em cada piquete de 0,5 ha nas safras de 2015/6 a 2018/9. As propriedades químicas foram determinadas seguindo Primavesi et al. (2005), sendo as medições de $\mathrm{pH}$ do solo foram feitas em $\mathrm{CaCl}_{2}$, e o $\mathrm{P}$ disponível foi avaliado pelo método da resina. Determinou-se a acidez potencial $(\mathrm{H}+\mathrm{Al})$ pelo método do $\mathrm{pH} \mathrm{SMP}$. Também foram medidos $\mathrm{K}^{+}, \mathrm{Ca}^{2+}, \mathrm{Mg}^{2+} \mathrm{e}$ $\mathrm{Al}^{3+}$ trocáveis. A capacidade de troca de cátions (CTC) e a saturação de base (\% V) foram calculadas.

A recomendação de calcário e fertilizantes foi estimada com base em Cantarella et al. (1996) e Bernardi et al. (2012), tendo sido utilizados os critérios: calagem (calcário dolomítico, PRNT 70\%) para aumentar a saturação de base para $70 \%$ para o milho e $60 \%$ para as pastagens, adubação com o P (superfosfato simples, $18 \% \mathrm{P}_{2} \mathrm{O}_{5}$ ) para aumentar o solo $\mathrm{P}$ para 15 $\mathrm{mg} \mathrm{dm}^{-3}$ ambas à taxa variável. As doses foram calculadas com o software descrito por Oliveira et al. (2010).

Cada piquete foi considerado uma "unidade de manejo", e a recomendação de calcário e fertilizante fosfatado foi calculada considerando a área de 0,5 ha, conforme descrito em Bernardi et al. (2017). A adubação com nitrogênio e potássio nas doses de 150 a $200 \mathrm{~kg} / \mathrm{ha}$ de $\mathrm{N}$ e $\mathrm{K}_{2} \mathrm{O}$ m doses uniformes e parceladas, na forma de formulação NPK 20-05-20. Anualmente realizou-se a coleta de amostras e análise de solo para calagem e adubação seguindo os resultados obtidos e critérios estabelecidos.

\section{Análise dos dados}

Inicialmente, todas as variáveis analisadas foram submetidas à análise exploratória calculando-se a média, desvio padrão, mínimo, máximo e os coeficientes de variação, assimetria e curtose (Tabela 1) nas quatro safras.

Os dados georreferenciados das recomendações de calagem e adubação com $\mathrm{P}$ foram interpolados pelo método do inverso da distância ponderada (IDW), calculados com a equação 1 :

$$
\mathrm{Xp}=\frac{\sum_{\mathrm{i}=1}^{\mathrm{n}}\left(\frac{1}{\mathrm{~d}_{\mathrm{i}}{ }^{2}} \mathrm{X}_{\mathrm{i}}\right)}{\sum_{\mathrm{i}=1}^{\mathrm{n}}\left(\frac{1}{\mathrm{~d}_{\mathrm{i}}{ }^{2}}\right)}
$$

Onde,

$\mathrm{X}_{\mathrm{p}}$ : variável interpolada; 
$\mathrm{X}_{\mathrm{i}}$ : valor da variável da i-ésima localidade vizinha;

$\mathrm{d}_{\mathrm{i}}$ : distância euclidiana entre o i-ésimo ponto de vizinhança e o ponto amostrado.
Os mapas de contorno (Figura 2) das necessidades de calcário e superfosfato simples (SSP) foram obtidos com o software ArcGIS 10.1 (ESRI, 2009).

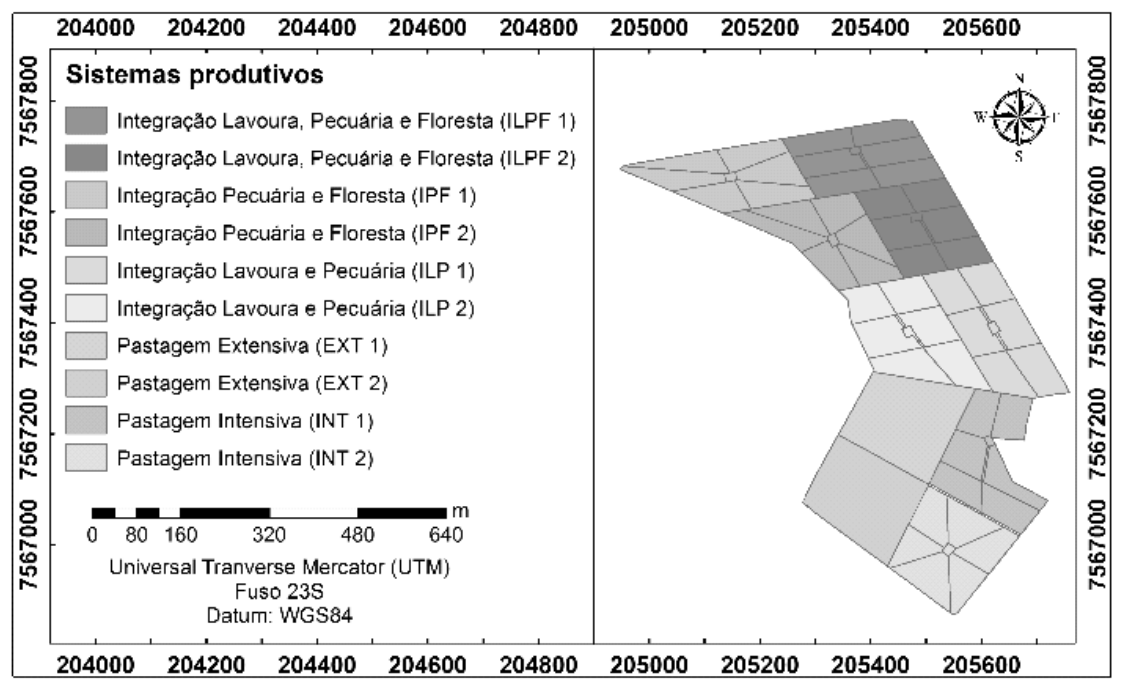

Figura 1: Localização da área de estudo, o sistema ILPF da Embrapa Pecuária Sudeste, em São Carlos - SP.

\section{RESULTADOS E DISCUSSÃO}

Os parâmetros estatísticos dos atributos do solo $\mathrm{pH}_{\mathrm{CaCl} 2}, \mathrm{P}_{\text {resina }}$, capacidade de troca de cátions (CTC), saturação por bases (V) e recomendações de calcário e fertilizante fosfatado estão apresentados na Tabela 1. Foram analisados os parâmetros estatísticos: média, desvio padrão, coeficiente de variação, valor mínimo, valor máximo, assimetria e curtose para verificar a existência de uma tendência central e dispersão de dados.

$\mathrm{O}$ coeficiente de variação $(\mathrm{CV}$ é considerado o primeiro indicador da heterogeneidade dos dados. Dessa forma, pode-se afirmar que o $\mathrm{pH}$ do solo é a propriedade do solo com menor variabilidade, com um coeficiente de variação abaixo de $10 \%$ nas quatro safras analisadas. A CTC e V\% representaram propriedades do solo com variabilidade média (CV 10 a 30\%). O teor de $\mathrm{P}$ foi a propriedade do solo com maior variabilidade, considerado muito altos (> $30 \%$ ). As tendências na variação dos atributos do solo obtidas neste estudo são consistentes com as observadas por Trotter et al. (2014) e Bernardi et al. (2016, 2017 e 2018) para os mesmos parâmetros do solo.

Em um conjunto de dados que apresente distribuição normal, os valores para os coeficientes de assimetria e curtose estarão entre zero e três (CARVALHO et al., 2000). Para a maioria das variáveis estudadas houve distribuição normal, conforme indicado pelos coeficientes de assimetria e curtose (Tabela 1). A exceção foi $\mathrm{O} \quad \mathrm{P}$ nas duas primeiras safras analisadas. Nas duas últimas, por efeito da correção dos níveis deste nutriente no solo os valores estavam mais próximos da distribuição normal. 
Tabela 1. Estatística descritiva de atributos do solo $\mathrm{pH}_{\mathrm{CaCl}}$, $\mathrm{P}_{\text {resina, }}$, capacidade de troca de cátions (CTC), saturação por bases (V) e recomendações de calcário e fertilizante fosfatado de um ILPF em 04 safras em São Carlos - SP.

\begin{tabular}{|c|c|c|c|c|c|c|c|}
\hline \multirow{2}{*}{ Safra } & \multirow{2}{*}{$\begin{array}{l}\text { Parâmetros } \\
\text { Estatísticos }\end{array}$} & \multirow{2}{*}{$\begin{array}{c}\mathbf{p H} \\
\left(\mathrm{CaCl}_{2}\right)\end{array}$} & \multirow{2}{*}{$\begin{array}{c}P_{\text {resina }} \\
\left(\mathbf{m g} / \mathbf{d m}^{3}\right)\end{array}$} & \multirow{2}{*}{$\begin{array}{c}\text { CTC } \\
\left(\mathrm{mmol}_{\mathrm{c}} / \mathrm{dm}^{3}\right) \\
\end{array}$} & \multirow{2}{*}{$\begin{array}{l}V \\
(\%)\end{array}$} & Calagem & SSP \\
\hline & & & & & & \multicolumn{2}{|c|}{$(\mathrm{kg} / \mathrm{ha})$} \\
\hline \multirow{8}{*}{$2015 / 6$} & $\mu$ & 4,8 & 8,1 & 58,3 & 45,6 & $1.090,0$ & 549,0 \\
\hline & $\sigma$ & 0,2 & 5,0 & 6,9 & 8,7 & 660,0 & 243,2 \\
\hline & Mínimo & 4,3 & 1,0 & 45,0 & 28,0 & 0,0 & 0,0 \\
\hline & Máximo & 5,4 & 26,3 & 77,0 & 66,0 & $2.500,0$ & 950,0 \\
\hline & CV (\%) & 3,9 & 61,0 & 11,8 & 19,0 & 60,6 & 44,3 \\
\hline & Curtose & 2,13 & 3,86 & 0,05 & ${ }^{-}, 16$ & $-0,76$ & 0,27 \\
\hline & Assimetria & 0,59 & 1,73 & 0,26 & 0,31 & $-0,12$ & $-0,81$ \\
\hline & $\mathbf{N}$ & 50 & 50 & 50 & 50 & 50 & 50 \\
\hline \multirow{8}{*}{$2016 / 7$} & $\mu$ & 5,1 & 10,2 & 61,2 & 60,0 & 410,0 & 165,0 \\
\hline & $\sigma$ & 0,2 & 6,3 & 5,3 & 7,7 & 459,5 & 167,9 \\
\hline & Mínimo & 4,7 & 3,0 & 55,0 & 47,0 & 0,0 & 0,0 \\
\hline & Máximo & 5,8 & 40,0 & 77,0 & 81,0 & $1.500,0$ & 500,0 \\
\hline & CV (\%) & 4,5 & 61,3 & 8,7 & 12,8 & 112,1 & 101,8 \\
\hline & Curtose & 0,33 & 9,27 & 1,28 & $\begin{array}{c}- \\
0,15\end{array}$ & $-0,69$ & $-1,43$ \\
\hline & Assimetria & 0,54 & 2,48 & 1,41 & 0,56 & 0,70 & 0,35 \\
\hline & $\mathbf{N}$ & 50 & 50 & 50 & 50 & 50 & 50 \\
\hline \multirow{8}{*}{ 2017/8 } & $\mu$ & 5,2 & 13,0 & 63,5 & 55,9 & 672,0 & 124,5 \\
\hline & $\sigma$ & 0,2 & 6,4 & 5,8 & 6,2 & 609,1 & 146,1 \\
\hline & Mínimo & 4,7 & 3,0 & 53,0 & 45,0 & 0,0 & 0,0 \\
\hline & Máximo & 5,7 & 28,0 & 79,3 & 68,0 & $2.500,0$ & 450,0 \\
\hline & CV (\%) & 4,0 & 49,5 & 9,2 & 11,0 & 90,6 & 117,3 \\
\hline & Curtose & $-0,13$ & $-0,93$ & $-0,08$ & 0,71 & $-0,06$ & $-1,13$ \\
\hline & Assimetria & $-0,07$ & 0,31 & 0,43 & 0,12 & 0,64 & 0,63 \\
\hline & $\mathbf{N}$ & 50 & 50 & 50 & 50 & 50 & 50 \\
\hline \multirow{8}{*}{$2018 / 9$} & $\mu$ & 5,4 & 13,2 & 71,2 & 65,2 & 120,0 & 110,0 \\
\hline & $\sigma$ & 0,3 & 6,9 & 9,7 & 8,8 & 295,5 & 116,9 \\
\hline & Mínimo & 4,9 & 6,0 & 53,0 & 47,0 & 0,0 & 0,0 \\
\hline & Máximo & 6,0 & 34,0 & 92,0 & 81,0 & $1.500,0$ & 350,0 \\
\hline & CV (\%) & 4,7 & 52,4 & 13,6 & 13,5 & 246,3 & 106,3 \\
\hline & Curtose & $-0,10$ & 2,01 & $-0,58$ & $\begin{array}{c}- \\
1,09\end{array}$ & 10,05 & $-1,07$ \\
\hline & Assimetria & 0,20 & 1,56 & 0,35 & $\overline{-}, 04$ & 2,98 & 0,58 \\
\hline & $\mathbf{N}$ & 50 & 50 & 50 & 50 & 50 & 50 \\
\hline
\end{tabular}

Os resultados da análise química de solo determinam o estoque de nutrientes no solo e os limitantes químicos no momento anterior ao plantio, possibilitando a recomendação de correção e adubação, bem como monitorar e avaliar periodicamente o balanço dos nutrientes no solo (CANTARELLA et al, 2002; BERNARDI et al., 2012). Comparando-se os resultados obtidos nas quatro safras indicaram que houve uma melhoria geral dos parâmetros analisados, e indicando que está havendo uma melhoria da fertilidade solo (Tabela 1).

$\mathrm{O}$ pH do solo é uma das propriedades químicas que mais influencia a disponibilidade de nutrientes para as plantas. No geral, os macronutrientes N, P, 
$\mathrm{K}, \mathrm{Ca}, \mathrm{Mg}$ e $\mathrm{S}$ têm sua disponibilidade aumentada com $\mathrm{pH}$ próximo à neutralidade. Para valores de $\mathrm{pH}$ inferiores a 5,5 aumentam a disponibilidade dos micronutrientes $\mathrm{Cu}, \mathrm{Fe}, \mathrm{Mn}$ e $\mathrm{Zn}$, mas acidez excessiva pode também aumentar a atividade de elementos potencialmente fitotóxicos, como Al e Mn (RAIJ, 1991). No Ano 1, os valores de $\mathrm{pH}_{\mathrm{CaCl} 2}$ (Tabela 1) estavam predominantemente na classe alta, com o decorrer das safras passaram para classe média ( $\mathrm{pH}$ entre 5,1 e 5,5) de acordo com os critérios de Raij et al. (1996). Esta redução da acidez do solo reflete o efeito positivo da calagem para os sistemas ILPF, sendo efeito similar ao já demonstrado por Caires et al. (1998), Luz et al. (2001), Rheinheimer et al. (2000) e Cantarella et al. (2002).

O macronutriente $\mathrm{P}$ é fundamental no desenvolvimento radicular e perfilhamento das gramíneas forrageiras, e sua deficiência limitar a capacidade produtiva das pastagens (MACEDO, 2004) Os baixos níveis de $\mathrm{P}$ no solo é um dos principais problemas no estabelecimento e na manutenção de pastagens nos solos tropicais (BERNARDI et al., 2012). Além da deficiência desse macronutriente, os solos tropicais têm uma alta capacidade de adsorção do $\mathrm{P}$ devido à acidez e altos teores de óxidos de $\mathrm{Fe}$ e $\mathrm{Al}$ (RAIJ, 1991). Por isso a adubação fosfatada é fundamental em sistemas de exploração extensivo ou intensivo (MACEDO, 2004), para garantir altas respostas da planta forrageira, principalmente quando são aplicados níveis elevados de nitrogênio (CANTARELLA et al., 2002) como neste sistema em estudo. Os valores de P (Tabela 1), seguindo a classificação de Raij et al. (1996) estavam na classe baixa (de 6 a 12 $\mathrm{mg} / \mathrm{dm}^{3}$ ) antes do início do programa de adubação a taxa variável, e gradualmente passaram para a classe média (de 12 a 30 $\mathrm{mg} / \mathrm{dm}^{3}$ ) após a aplicação de superfosfato simples. Os resultados comprovam que, em pastagens já formadas, a adição de $\mathrm{P}$ pode garantir níveis adequado no solo e produtividade elevados da forrageira (CANTARELLA et al., 2002).
Dentre as variáveis que definem a fertilidade do solo, destaca-se a capacidade de troca catiônica (CTC), que é a quantidade total de cátions retidos à superfície das partículas de solo (RAIJ, 1991). A CTC é função da mineralogia do solo, da matéria orgânica e do $\mathrm{pH}$, e promove a retenção de cátions $(\mathrm{K}, \mathrm{Ca} \mathrm{e}$ $\mathrm{Mg}$ ) e diminui as perdas por lixiviação destes nutrientes. Os valores da capacidade de troca catiônica (CTC, Tabela 1), foram alterados nas observações dos 4 anos, porém permanecem predominantemente na classe média (43 a $86 \mathrm{mmol}_{\mathrm{d}} / \mathrm{dm}^{3}$ ) de acordo com a classificação proposta por Alvarez Venegas et al (1999).

$\mathrm{O}$ valor da saturação por bases (V\%) indica a porcentagem das cargas negativas ocupadas por $\mathrm{K}, \mathrm{Ca}, \mathrm{Mg}$, $\mathrm{Na}$, e passíveis de troca a $\mathrm{pH}$ 7,0 quando comparado com os pontos ocupados por $\mathrm{H}+\mathrm{Al}$ (RAIJ, 1991). Solos com V\% muito baixa requerem maiores quantidades de calcário para elevar a saturação de bases. Os valores de V\% sempre foram mantidos mais altos nas áreas de plantio de milho, por ser uma cultura mais exigente (CANTARELLA et al., 1996). Já valores de V\% na área de pastagem foram mantidos em $60 \%$.

Os valores da saturação por bases (V\%, Tabela 1), seguiram a mesma tendência da CTC, e confirmam os resultados benéficos da calagem na redução da acidez potencial e elevação das bases do solo. Considerando a classificação proposta por Raij et al. (1996) os valores da V\% no início do estudo estavam na classe baixa (entre 26 e $50 \%$ ), e com a aplicação de calcário a taxas variáveis elevou-se para a classe média (entre 51 e 70\%). Mas, houve valores máximos dentro da classe considerada alta (de 71 a 90\%)

Os resultados obtidos no $4^{\circ}$. ano de avaliação indicaram que os parâmetros de fertilidade do solo estão mais homogêneos, como também havia demonstrado Richart et al (2016) e Bernardi et al. (2018).

A tecnologia de aplicação a taxa variável (BERNARDI et al., 2004; 
RESENDE et al., 2014), considerando os piquetes como unidades mínimas de manejo (BERNARDI et al., 2017) foi implementada a partir da $1^{\text {a }}$ safra $(2015 / 6)$ e foi utilizada nas safras seguintes $(2016 / 7$, 2017/8, e 2018/9). As interpolações dos mapas de recomendação de calcário e fósforo foram obtidas pelo método IDW e estão apresentadas nas Figuras 2 e 3 respectivamente. Por meio da geração destes mapas foi possível a aplicação dos insumos a taxa variada nas unidades mínimas de manejo (BERNARDI et al., 2017). Serrano et al. (2011), Trotter et al. (2014) e Bernardi et al. (2016, 2018) também estabeleceram com sucesso mapas de recomendação de fertilizantes com base
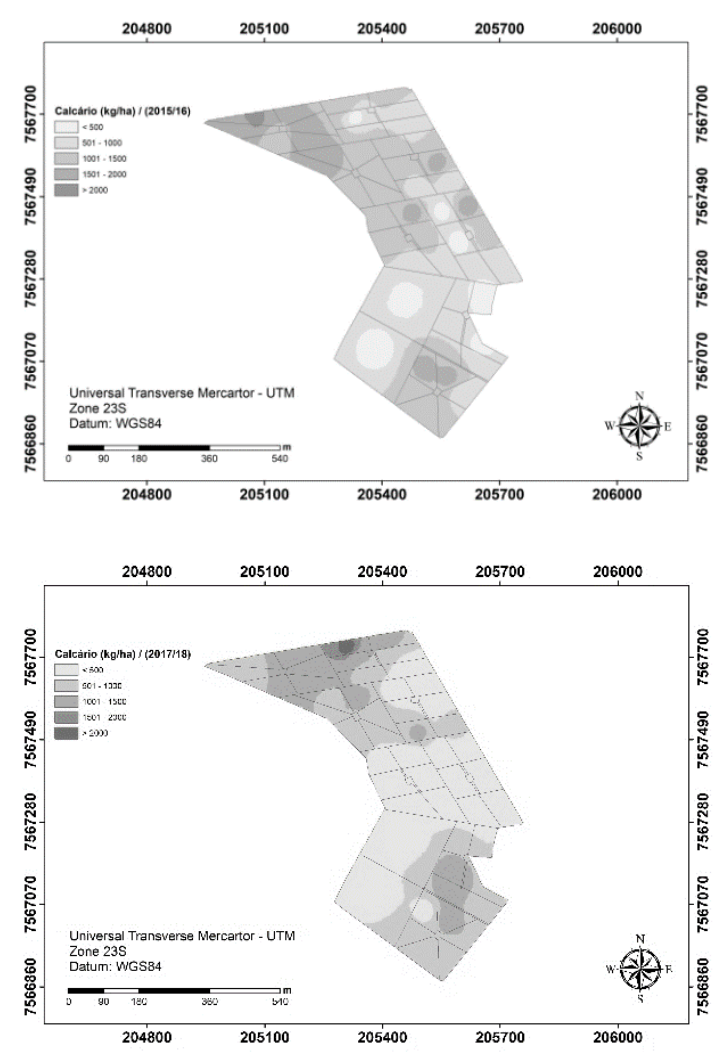

na disponibilidade de nutrientes do solo em sistemas de pastagem.

Com isso, foi possível verificar-se a gradual melhoria da fertilidade do solo ao longo dos 4 anos de avaliação (Tabela 1). A melhoria nos atributos químicos do solo com a aplicação superficial de calcário como observados neste trabalho seguem a tendência observada por Caires et al. (1998) e Rheinheimer et al., 2000). A menor variação na disponibilidade de $\mathrm{P}$ do solo, indicada pelas doses de fertilizante fosfatado também tiveram a variabilidade espacial reduzida, como também havia sido demostrado por Serrano et al. (2011).

A

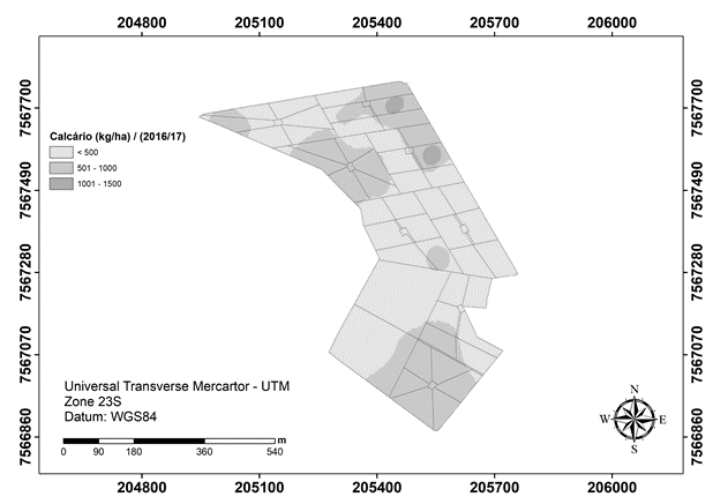

C

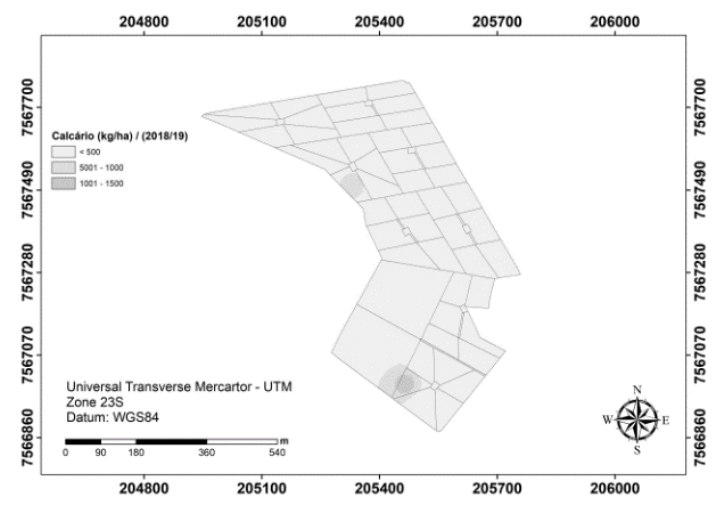

Figura 2. Mapas interpolados de aplicação de calcário nas safras 2015-16 (A), 2016-7 (B), 2017-18 (C), e 2018-19 (D) em sistema ILPF em São Carlos - SP.

As doses de calcário eram maiores no início do estudo (2015/6) e foram gradativamente reduzindo ao longo das quatro safras (Tabela 1 e Figura 2). Sendo que no último ano avaliado, a maior parte da área indicou necessidades de doses baixas de calcário (até $1 \mathrm{t} / \mathrm{ha}$ ) com alguns pontos chegando a 1,5 t/ha (Figura 2), e indicam que o solo foi sendo gradativamente corrigido.

A recomendação para adubação fosfatada na $1^{\text {a }}$ safra $(2015 / 6)$ indicava doses elevadas de superfosfato simples (> $500 \mathrm{~kg} / \mathrm{ha}$ ) em mais de $80 \%$ da área. Com a adubação a taxa variável, nas safras seguintes observou-se redução das doses 
necessárias, como menor variabilidade espacial das doses. Indicando, com isso que a prática da adubação com $\mathrm{P}$ a taxas variáveis estava sendo eficiente para correção da fertilidade do solo, a exemplo do que havia sido observado por Serrano et al. (2011).

Os mapas das Figuras 2 e 3 comprovam a melhoria da fertilidade pois indicam que no sistema ILPF, foi possível reduzir em 89 e $57 \%$ as quantidades totais de calcário e supersimples. As doses de totais aplicadas em 30ha variaram de 54,5 e 27,5 t/ha aplicadas em 2015, para 6,0 e 11,7 t/ha aplicados em 2018, respectivamente.
Os resultados obtidos confirmam que a aplicação a taxa variável destes insumos (calcário e adubo fosfatado) foram eficientes em corrigir o solo e torná-lo mais homogêneo. Entretanto, como destacaram Gillingham (2001) deve ser implementado um diagnóstico adequado dos fatores limitantes à produção das pastagens, pois o aumento das doses de fertilizantes em locais que o crescimento das pastagens é limitado por outros fatores (restrições físicas, reboleiras de pragas, doenças ou plantas daninhas, etc) que não a fertilidade do solo pode limitar a produtividade.

A
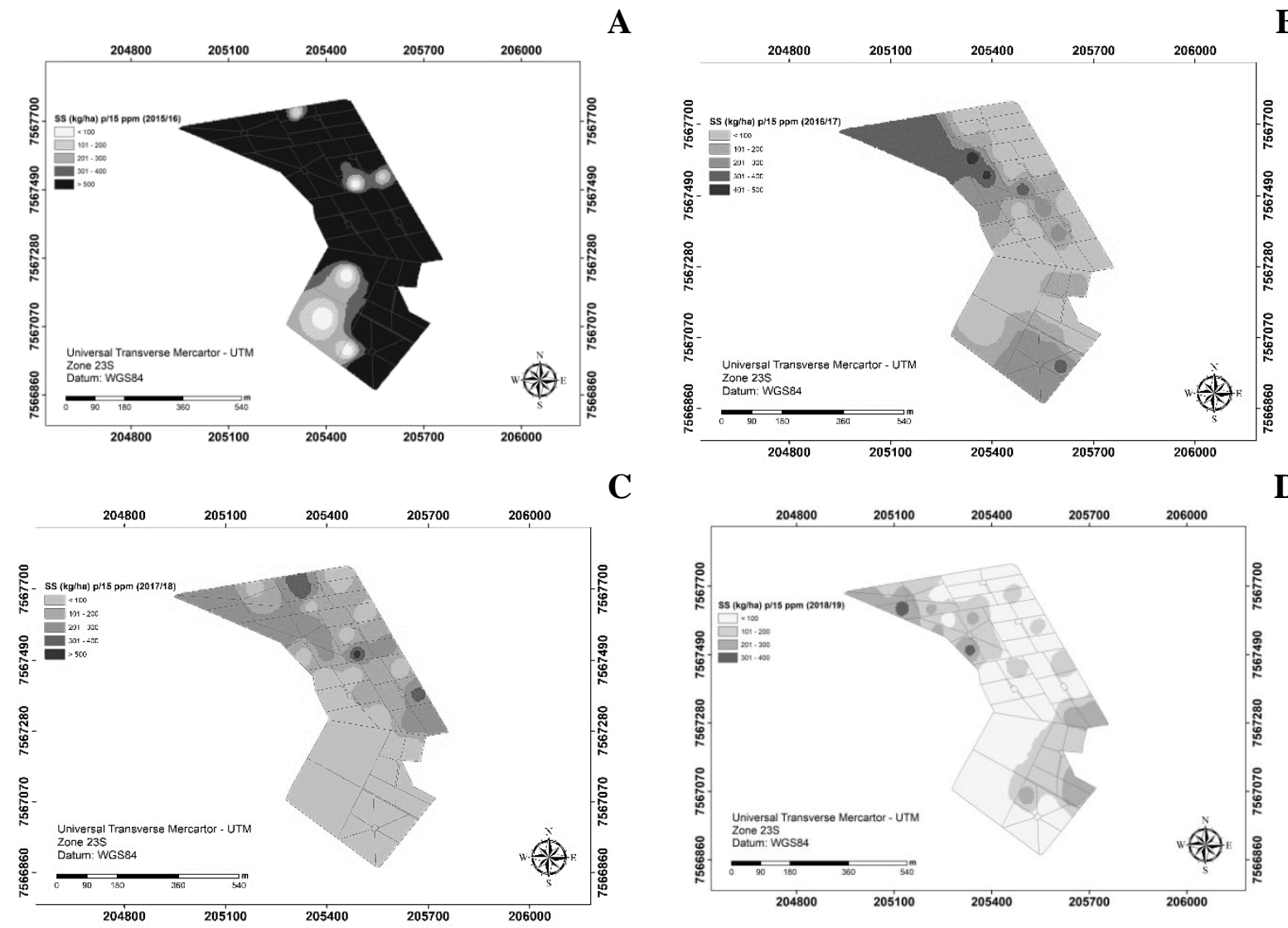

Figura 3. Mapas interpolados de aplicação de superfosfato simples nas safras 2015-16 (A), 2016-7 (B), 2017-18 (C), e 2018-19 (D) em sistema ILPF em São Carlos - SP. 


\section{CONCLUSÕES}

Os resultados mostraram que a análise espacial das necessidades de calagem e adubação podem fornecer ferramentas de gestão para o manejo de pastagens. E a tecnologia da aplicação de calcário e fertilizante fosfatado à taxa variável pode ser utilizada como ferramenta de correção e adubação do solo levando à maior homogeneidade dos atributos químicos do solo.

\section{AGRADECIMENTOS}

Ao International Potash Institute - IPI e Associação Rede ILPF pelo apoio financeiro na condução deste trabalho.

\section{REFERÊNCIAS}

ALVAREZ VENEGAS, V.H.; NOVAIS, R.F.; BARROS, N.F.; CANTARUTTI, R.B.; LOPES, A.S. Interpretação dos resultados das análises de solos. In: RIBEIRO, A.C.; GUIMARÃES, P.T.; ALVAREZ VENEGAS, V.H. (Ed.). Recomendações para o uso de corretivos e fertilizantes em Minas Gerais. Viçosa: UFV, 1999. p.25-32.

BERNARDI, A. C. C.; BETTIOL, G. M.; FERREIRA, R. P.; SANTOS, K. E. L.; RABELLO, L. M.; INAMASU, R. Y. Spatial variability em São Carlos - SP, Brasil of soil properties and yield of a grazed alfalfa pasture in Brazil. Precision agriculture, v. 17, p. 737-752, 2016.

BERNARDI, A. C. C.; BETTIOL, G. M.; GREGO, C. R.; ANDRADE, R. G.; RABELLO, L. M.; INAMASU, R. Y. Ferramentas de agricultura de precisão como auxílio ao manejo da fertilidade do solo. Cadernos de Ciência \& Tecnologia, v. 32, n. 1/2, p. 205-221, 2015.

BERNARDI, A. C. C.; BETTIOL, G. M.; MAZZUCO, G. G.; ESTEVES, S. N.; OLIVEIRA, P. P. A.; PEZZOPANE, J. R. M. Spatial variability of soil fertility in an integrated crop livestock forest system. Advances in Animal Biosciences, v. 8, n. 2, p. 590-593, 2017.

BERNARDI, A. C. C.; BUENO, J. O. A.; LAURENTI, N.; SANTOS, K. E. L.; ALVES, T. C. Efeito da calagem e fertilizantes aplicados à taxa variável nos atributos químicos do solo e custos de produção de pastagem de capim Tanzânia manejadas intensivamente. Brazilian Journal of Biosystems Engineering, v.12, n.4, p.368-382, 2018.

BERNARDI, A. C. C.; GIMENEZ, L. M; MACHADO, P. L. O. A.; SILVA, C. A. Aplicação de fertilizantes a taxas variáveis. In: MACHADO, P. L. O. A.; BERNARDI, A. C. C.; SIlvA, C. A. (Org.). Agricultura de precisão para o manejo da fertilidade do solo em sistema plantio direto. 1ed. Rio de Janeiro: Embrapa Solos, 2004. p. 153-164

BERNARDI, A. C. C.; OLIVEIRA, P. P. A.; PRIMAVESI, O. Soil fertility of tropical intensively managed forage system for grazing cattle in Brazil. In: WHALEN, J.K. Soil fertility improvement and integrated nutrient management - a global perspective. Rijeka, Croatia: Intechopen, 2012. p. 37-56. Disponível em:

http://www.intechopen.com/books/soilfertility-improvement-and-integratednutrient-management-a-globalperspective/soil-fertility-of-tropicalintensively-managed-forage-system-forgrazing-cattle-in-brazil.

CAIRES, F. E.; CHVEIRI, A. W.; MADRUGA, E. F.; FIGUEIREDO, A. Alterações de características químicas do 
solo e resposta da soja ao calcário e gesso aplicado na superfície em sistema de cultivo sem preparo do solo. Revista Brasileira de Ciência do Solo, v.22, p.2734, 1998.

CALDERANO FILHO, B., SANTOS, H.G., FONSECA, O.O.M., SANTOS, R.D., PRIMAVESI, O. E PRIMAVESI, A.C. 1998. Os solos da Fazenda Canchim, Centro de Pesquisa de Pecuária do Sudeste, São Carlos, SP: levantamento semidetalhado, propriedades e potenciais. Embrapa-CPPSE, São Carlos. Boletim de Pesquisa.

CANTARELLA, H.; CORREA, L.A.; PRIMAVESI, O.; PRIMAVESI, A.C. Fertilidade do solo em sistemas intensivos de manejo de pastagens. In: Anais do Simpósio sobre Manejo de Pastagens. Piracicaba: FEALQ. 2002. p.99-131.

CANTARELLA, H.; RAIJ, B. van; CAMARGO, C.E.O. Cereais. In: RAIJ, B. van; CANTARELLA, H.; QUAGGIO, J.A. FURLANI, A.M.C. (eds.) Recomendações de adubação e calagem para o Estado de São Paulo. Campinas, Instituto Agronômico, 1996. p.43-71.

CARVALHO, J. R. P.; SILVEIRA, P. M.; VIEIRA, S. R. Geoestatística na determinação da variabilidade espacial de características químicas do solo sob diferentes preparos. Pesquisa Agropecuária Brasileira, v.37, p.11511159, 2002.

GILLINGHAM, A. G. Precision management of fertiliser application to pasture. Proceedings of the Regional Institute, Geo-spatial Information in Agriculture Conference, 2001. Paper no. 534. p. 534-541. Disponível em: http://www.regional.org.au/au/gia/16/534g illingham.htm

INAMASU, R. Y.; BERNARDI, A. C. C.; VAZ, C. M. P.; NAIME, J. M.; QUEIROS, L. R.; RESENDE, A. V.; VILELA, M. de F.; JORGE, L. A. C.; BASSOI, L. H.; PEREZ, N. B.; FRAGALLE, E. P. Agricultura de precisão para a sustentabilidade de sistemas produtivos do agronegócio brasileiro. In: INAMASU, R. Y.; NAIME, J. M.; RESENDE, A. V.; BASSOI, L. H.; BERNARDI, A. C. C. (Ed.). Agricultura de precisão: um novo olhar. São Carlos: Embrapa Instrumentação, 2011. p. 14-26.

LUZ, P. H.; HERLING, V. R.; PETERNELLI, M.; BRAGA, G. J. Calagem e adubação no manejo intensivo do pastejo. In: EVANGELISTA, A.R.; SALES, E. C. J.; SIQUEIRA, G. R.; LIMA, J. A., eds., Anais do $2^{\circ}$ Simpósio de Forragicultura e Pastagens: temas em evidência. Lavras: Univ. Federal de Lavras, 2001. p.27-110.

MACEDO, M.C.M. Adubação fosfatada em pastagens cultivadas com ênfase na Região do cerrado. In: YAMADA, T.; ABDALA, S.R.S. (Eds.). Fósforo na agricultura brasileira. Piracicaba: Associação Brasileira para a Pesquisa da Potassa e do Fosfato, 2004. p.359-400.

OLIVEIRA, P. P. A.; BERNARDI, A. C. C.; SANTIAGO, R. R.; SILVA, R. F. Software adubapasto 1.0 para recomendação de calagem e adubação para sistemas de pastejo intensivo. São Carlos, SP: Embrapa Pecuária Sudeste, 2010. (Embrapa Pecuária Sudeste. Documentos, 98).

PRIMAVESI, A. C.; ANDRADE, A. G.; ALVES, B. J. R.; ROSSO, C.; BATISTA, E. M.; PRATES, H. T.; ORTIZ, F. R.; MELLO, J.; FERRAZ, M. R.; LINHARES, N. W.; MACHADO, P. L. O. A.; MOELLER, R.; ALVES, R. C. S.; SILVA, W. M. Métodos de análise de solo. In: NOGUEIRA, A. R. A.; SOUZA, G. B. Manual de laboratórios: Solo, água, nutrição vegetal, nutrição animal e alimentos. São Carlos: Embrapa Pecuária Sudeste, 2005. p. 67-130.

RAIJ, B. van. Fertilidade do solo e adubação. Piracicaba: Agronômica Ceres; Associação Brasileira para Pesquisa da Potassa e do Fosfato, 1991. 343p.

RAIJ, B. van; QUAGGIO, J.A.; 
CANTARELLA, H.; ABREU, C.A. Interpretação de resultados de análise de solo. In: RAIJ, B. van; CANTARELLA, H.; QUAGGIO, J.A. FURLANI, A.M.C. (eds.) Recomendações de adubação e calagem para o Estado de São Paulo. Campinas, Instituto Agronômico, 1996. p.8-13.

RESENDE, A. V.; SHIRATSUCHI, L. S.; COELHO, A. M.; CORAZZA, E. J.; VILELA, M. F.; INAMASU, R. Y.; BERNARDI, A. C. C.; BASSOI, L. H.; NAIME, J. M. Agricultura de precisão no Brasil: avanços e impactos no manejo e na conservação do solo, na sustentabilidade e na segurança alimentar. In: LEITE, L. F. C.; MACIEL, G. A.; ARAÚJO, A. S. F. (Ed.). Agricultura conservacionista no Brasil. Brasília, DF: Embrapa, 2014. p. 468-488.

RHEINHEIMER, D.S.; SANTOS, E.J.S.; KAMINSKI, J.; BORTOLUZZI, E.E. \& GATIBONI, L.C. Alterações de atributos do solo pela calagem superficial e incorporada a partir de pastagem natural.

Revista Brasileira de Ciência do Solo, v.24, p.795-805, 2000.

RICHART, A.; PICCIN, A. L.; KONOPATZKI, M. R. S.; KAEFER, K. A. C.; MORATELLI, G.; KtAEFER, J. E.; ECCO, M. Análise espaço-temporal de atributos químicos do solo influenciados pela aplicação de calcário de cloreto de potássio em taxa variável. Scientia Agraria Paranaensis, v. 15, n. 4, p. 391400, 2016.

ROLIM, G. D. S.; CAMARGO, M. B. P. D.; LANIA, D. G.; MORAES, J. F. L. D. Climatic classification of Köppen and Thornthwaite sistems and their applicability in the determination of agroclimatic zonning for the state of São Paulo, Brazil. Bragantia, v.66, n.4, p.711720, 2007.

SANTOS, K. E. L.; BERNARDI, A. C. de C.; BETTIOL, G. M.; CRESTANA, S. Geoestatística e geoprocessamento na tomada de decisão do uso de insumos em uma pastagem. Brazilian Journal of Biosystems Engineering, v. 11, n. 3, p. 294-307, 2017.

SERRANO, J. M.; PEÇA, J. O.; SILVA, J. R. M.; SHAHIDIAN, S. Spatial and temporal stability of soil phosphate concentration and pasture dry matter yield. Precision Agriculture, v. 12, n. 2, p. 214232, 2011.]

TROTTER, M.; GUPPY, C.; HALING, R.; TROTTER, T.; EDWARDS, C.; LAMB, D. Spatial variability in $\mathrm{pH}$ and key soil nutrients: is this an opportunity to increase fertiliser and lime-use efficiency in grazing systems? Crop and Pasture Science, v.65, p. 817-827, 2014. 